\title{
Design of On chip Spiral Inductors for Millimeter Wave Frequency Synthesizers
}

\author{
Nithin M, Member, IEEE, Harish M Kittur, Senior Member, IEEE
}

\begin{abstract}
The energy storing element, inductor plays a vital role in CMOS based high frequency integrated circuits, especially in signal generation and impedance matching blocks.An on chip inductor is considered as a critical component because its performance directly impacts the associated circuitry when it is used as a load device or as a matching element. Out of the various requirements of an inductor which resides inside a chip, the inductance value,quality factor and self resonance frequency with smaller area is often preferred. This paper focuses on the lumped model of inductors for high frequency circuits working in the Millimeter wave region from $30 \mathrm{GHz}$ to $300 \mathrm{GHz}$. For millimeter wave oscillators, inductance value in the range of pico Henry are essential and hence a complete model of an inductor is presented. Using electromagnetic simulator SONNET, all the parameters are extracted. The extracted model is used in the design of an LC Oscillator for millimeter wave band. A Q factor of 26 is achieved for an inductor value close to $153 \mathrm{pH}$ at 60 GHz.The circuits employing this inductor shows promising results when simulated using $45 \mathrm{~nm}$ CMOS pdks.
\end{abstract}

Keywords - Spiral inductors, Millimeter wave oscillators, Quality factor.

\section{INTRODUCTION}

I NTERNET of Things (IoT) and Internet of Everythings (IoE) are the need of the hour. They are the key enablers in connecting people across the globe in the 5th generation cellular network. As the lower Giga Hertz frequencies in the spectrum are overcrowded and the demands for technology driven wireless devices are becoming more and more, efforts and researches are happening for past one decade to move to the higher ends of the spectrum. In relation to this Milli-Meter Wave (MMW) Communication is often heard and MMW circuits are designed to cater the needs of a $5 \mathrm{G}$ cellular network.

For any wireless communication system, transceivers are the fundamental and the crucial block which facilitates transmission and reception. Among the various circuit blocks in a transceiver system, oscillators play a key role in the frequency band in which the system works. Oscillators are circuits which generate a periodic signal in its steady state. Voltage Contolled Oscillators (VCOs)[1] are widely used for frequency synthesis purpose in a transceiver. In high frequency integrated circuits, oscillators are designed using LC based tank circuits which uses on chip inductors.

The rest of the paper is organized as follows. Section II brings the background of the work in the light of high fre-

Nithin $M$ is with School of Electronics Engineering, VIT, Vellore, Tamil Nadu, India as a Research Scholar and with Department of ECE, RV College of Engineering, Bengaluru, India as an Assistant Professor, email:nithinm@rvce.edu.in

Harish M Kittur is with School of Electronics Engineering, VIT, Vellore, Tamil Nadu, India as Professor and Dean. quency oscillators.Section III describes the background of on chip inductors and its geometrical design parameters. Section IV brings the design problem at MMW region and proposes an algorithm for optimization. Section $\mathrm{V}$ discusses modeling of spiral inductors followed by optimization using an EM simulator in section VI. Section VII compares the performance parameters of oscillator circuits designed at MMW region followed by conclusion in section VIII.

\section{BACKGROUND}

Over the years, many architectures of oscillators have been proposed. Oscillators can be classified as two main categories [2], one with resonator and the other without resonator. Generally, oscillators need complex conjugate poles for its sustained oscillations. Resonator less oscillators are designed using one type of reactive element, either inductors or capacitors and resistor or a transconductance device. In order to make the resonator less oscillators work, proper feedback should be ensured. Ring oscillator is a popular resonator less oscillator, which usually consists of a series of inverting stages in cascade and by connecting the output of last inverter to the input of the first inverter. Figure 1 shows the classification of high frequency oscillators.

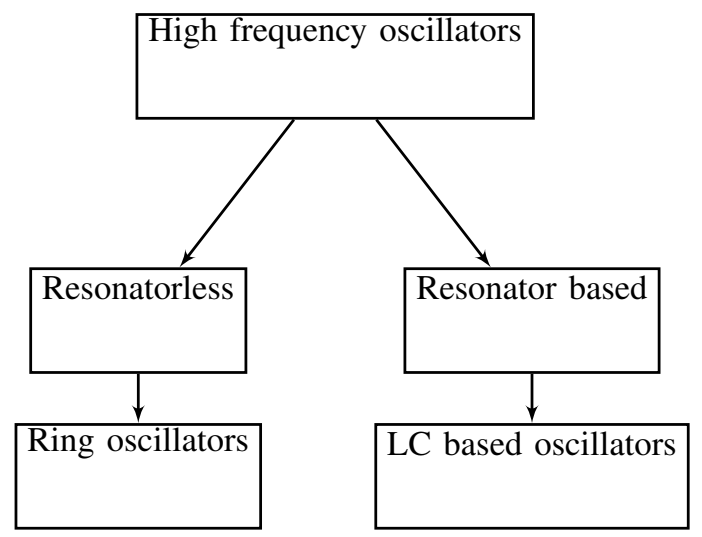

Fig. 1. Classification of high frequency oscillators

The ring oscillators reported in Jean Olivier Plouchart (2004) in bulk CMOS processes [3] are limited with their frequency of oscillation and hardly reaches the millimeter wave regime. These reasons made ring oscillators not so popular for MMW applications. But some of the later advances shows that [4] Marius Voicu (2013) the performance of ring oscillators are comparable to LC based oscillators especially at frequencies beyond $60 \mathrm{GHz}$.

Resonator based oscillators are designed using resonators or tanks which has inherent complex poles for sustaining 
oscillations. Resonators use both reactive elements - inductors and capacitors and therefore known as LC oscillators.

\section{A. LC tank Integrated oscillators}

[5]

An ideal LC tank can be picturized as shown in Fig 2.

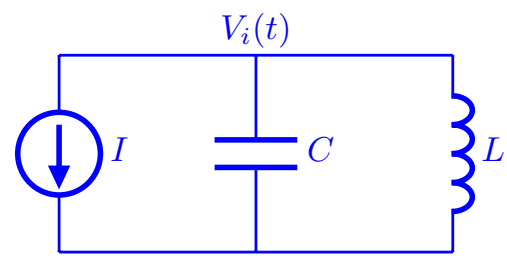

Fig. 2. Ideal LC tank circuit

Since an ideal LC tank is lossless, it will sustain oscillations if excited using a current source. The energy storing elements will periodically exchange their energy indefinitely and a periodic waveform will be sustained. But in reality, the tank is lossy and losses can be modelled using a parallel resistance $R_{p}$, across tank as shown in Fig 3.

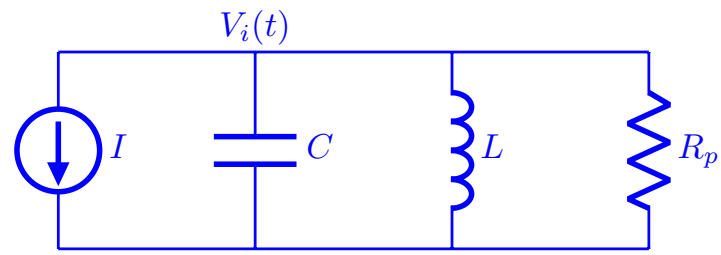

Fig. 3. LC tank circuit with losses

This dissipative element $R_{p}$ will cause the oscillations to die out. So, the key idea and the conventional approach is to replenish the losses in the tank using a negative resistance $-R_{p}$, which can in turn sustain the oscillations by cancelling the losses in $R_{p}$ as shown in Fig 4.

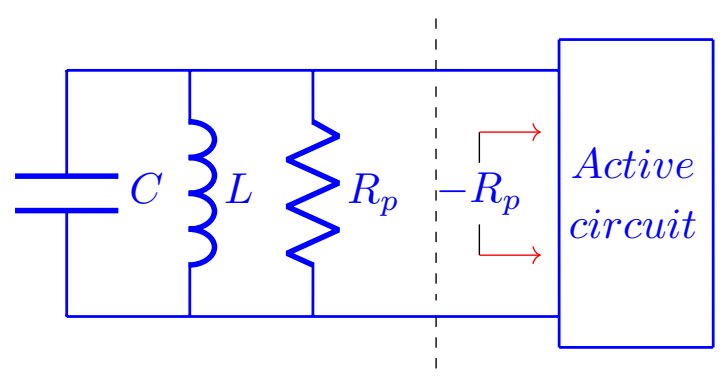

Fig. 4. One port view of oscillators

Therefore, oscillations will occur with a frequency of

$$
f=\frac{1}{2 \pi \sqrt{L C}}
$$

Usually the negative resistance is achieved using a transistor as an active circuit across the lossy tank as explained in [?]. The condition for sustaining oscillations in such circuit is given by

$$
\left|g_{m} R_{p}\right| \geq 1
$$

\section{B. Popular LC oscillator architectures}

The popular LC oscillator topologies are summarized in this section. The most common topology is cross coupled architecture with a current source to ground.This has the smallest sensitivity to noise on the ground line and highest sensitivity to supply pushing [6]. The symmetrical waveforms of this structure make it with a lower flicker noise upconversion than its current source to supply counterpart. Since the inductors are connected to supply voltage, these structures have a signal swing of up to twice the supply voltage on each node. This signal maximization leads to phase noise minimization. The high signal swing can cause hot electron effects in MOSFETs and may even cause gate oxide breakdown. Therefore, these structures are suitable for low power supply operation. Both the structures are shown in Fig 5.
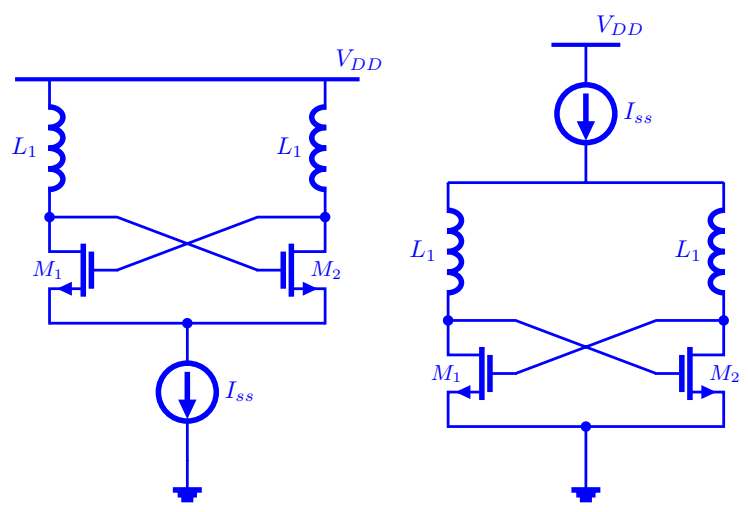

Fig. 5. Cross coupled oscillator using NMOS cores with current source to ground and supply

The popular oscillators use inductive loads to generate high frequency oscillations. Therefore the next section discusses the methods to incorporate inductors within a chip, its modeling and parameter extraction.

\section{ON CHIP INDUCTORS - BACKGROUND}

On chip inductors are of high demand in high frequency RF circuits. Spiral inductor is a very popular one in this category. They are realised on chip by putting metal traces on silicon substrate with the aid of multiple interconnects.[7] One of the widely used planar topology is a square structure based spiral as shown in Figure 6. The cross section with magnetic lines are shown in Figure 7. There will be linking between the flux lines through the centre and the flux in the turns of the spiral. The magnetic flux $\Psi(\mathrm{Wb})$ in the spiral structure can be expressed as

$$
\begin{aligned}
& \Psi=\oint B . d S \\
& =\mu \oint H . d S
\end{aligned}
$$

where $\mathrm{B}$ is magnetic flux density in tesla, $\mathrm{H}$ is magnetic field density in $\mathrm{A} / \mathrm{m}$ and $\mathrm{dS}$ is area in $\mathrm{m}^{2}$. The self inductance of the coil is given by

$$
L=\frac{\Psi}{I}
$$




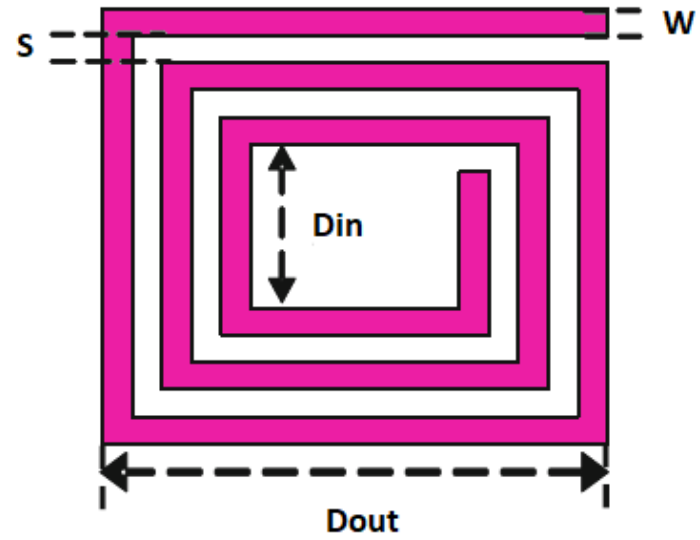

Fig. 6. Square Spiral structure

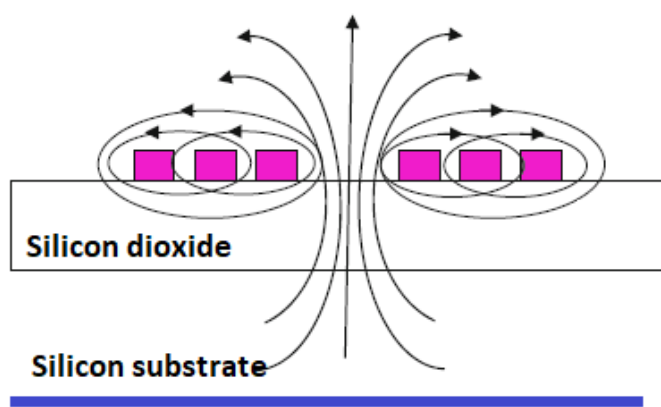

Fig. 7. Square Spiral structure - cross section

Energy will be stored in the inductor from the voltage applied and is given by

$$
V=\frac{d \Psi}{d t}=L \times \frac{d i}{d t}
$$

The magnetic interaction between two currents results in mutual inductance and that will be added to the self inductance.Therefore the total inductance is calculated as

$$
L_{\text {total }}=L_{\text {self }}+M_{+}+M_{-}
$$

where $L_{\text {self }}$ is the total self inductance sum of all the segments of the structure and $M_{+}$and $M_{-}$are the mutual inductances sum of all the segments.

Apart from square spirals [8], various other planar structures are also used and reported. Some of them are meander, circular and octagonal geometries. Figure 8 shows commonly used octagonal spiral structures. They are generally characterized with a set of geometrical design parameters like

1) Outer and inner diameter, $D_{\text {out }}$ and $D_{\text {in }}$

2) Line width or spiral track width, W

3) Number of turns, $\mathrm{N}$

4) Spiral track spacing, $\mathrm{S}$

The inductor also considers the following figures of merit like

1) Q factor or Quality factor

2) Maximum frequency $f_{\max }$ where $Q_{\max }$ is achieved

3) Self resonance frequency $f_{\text {self }}$
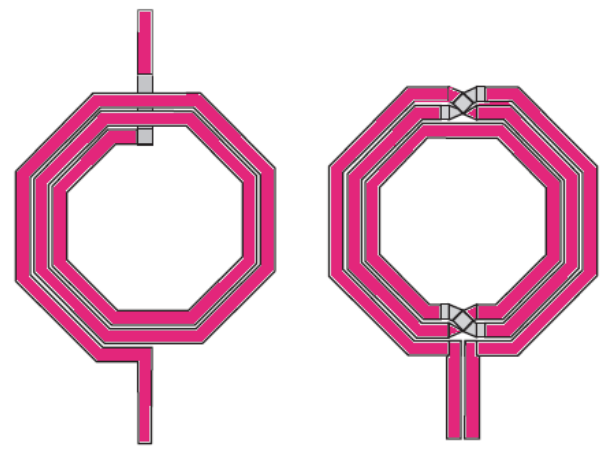

Fig. 8. Various on chip Spiral inductor structures

\section{SPIRAL INDUCTOR DESIGN PROBLEM AT MMW REGION}

In order to obtain the inductance value required at the millimeter wave region, the layout parameters to be optimized. Typically the required optimization is formulated to get maximum $\mathrm{Q}$ factor at the required frequency by choosing the optimum geometries [9]. It can be written as

\section{maximizeQfactor fromthegeometries}

$$
\begin{gathered}
L \leq L_{\text {desired }} \\
N_{\text {min }} \leq N \leq N_{\max } \\
W_{\text {min }} \leq W \leq W_{\max } \\
S_{\min } \leq S \leq S_{\max } \\
D_{\min } \leq D \leq D_{\max }
\end{gathered}
$$

The optimization variables are the dimensions of the spiral structure. These variables are dependent on each other by the relation

$$
D_{\text {out }}=D_{\text {in }}+2 \times W \times N+2 \times S \times(N-1)
$$

Several combinations of the geometrical variables may give the desired value of inductance and a set of inductance values with varying parameters may give same area as well. The following algorithm is proposed to optimize the spiral inductor structure by estimating the bounds on the geometrical variables.

The self inductance of a spiral segment is given as

$$
L=0.002 l\left(\ln \frac{2 l}{W+t}+0.50049+\frac{W+t}{3 l}\right)
$$

where 1 and $t$ are the length and thickness of the spiral conductor.

Two equal length conductors will have mutual inductance given by

$$
M=2 \times l \times P
$$

$\mathrm{P}$, the $L_{\text {mutual }}$ parameter is given as

$$
P=\ln \left[\frac{l}{G D}+\sqrt{1+\frac{l^{2}}{G D^{2}}}\right]-\sqrt{1+\frac{G D^{2}}{l^{2}}}+\frac{G D}{l}
$$

where GD is the mean distance of the geometrical structure. 


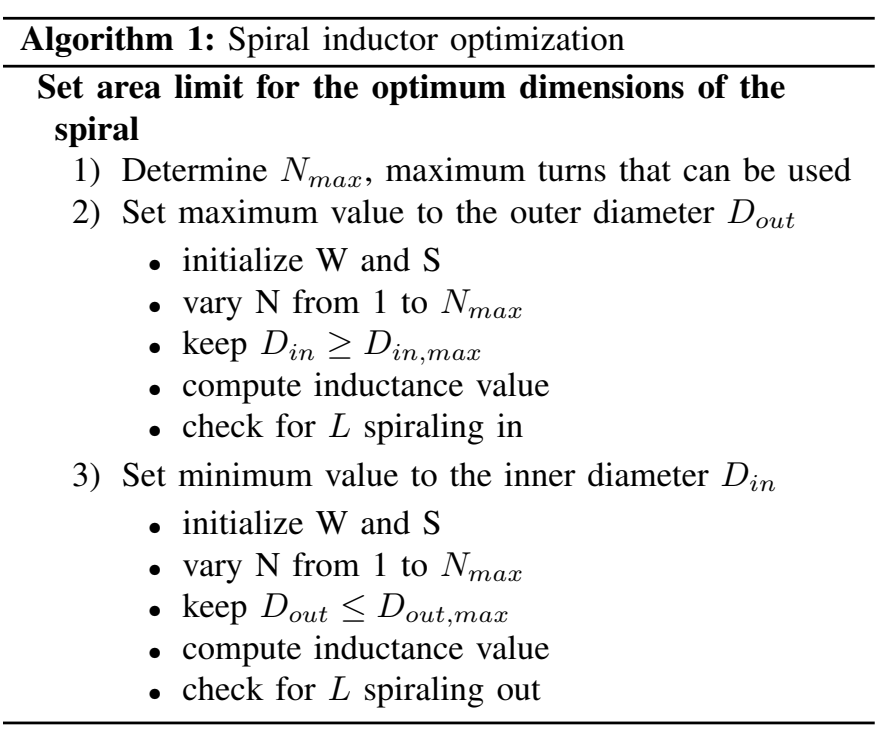

\section{Modeling OF Spiral Inductors}

A well known model based on lumped elements is used for accurate optmization. It consists of inductor and the parasitics associated with it as shown in Figure 9.

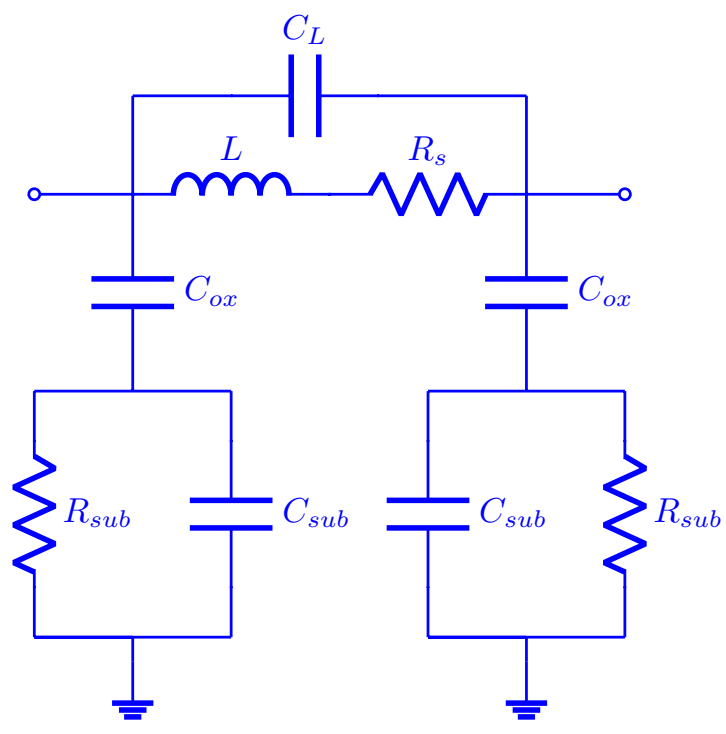

Fig. 9. Spiral inductor - Equivalent Circuit

As represented in the equivalent circuit, a set of passive elements are used to describe the electrical beheviour of the inductor.The elemental values of the model can be expressed as follows.

Series resistance $R_{s}$ is given by

$$
R_{s}=\frac{\rho \times l}{W \delta\left(1-e^{t / \delta}\right)}
$$

The spiral structure overlap and the crosstalk in the turns (adjacent) is modeled by a capacitance $C_{s}$ given by

$$
C_{L}=n W^{2} \frac{\epsilon_{o x}}{t_{o x}}
$$

The parasitics in the substrate and oxide are represented by $R_{s u b}$ and $C_{\text {sub }}$ respectively as

$$
\begin{aligned}
C_{s u b} & =C_{o x} \frac{1+\omega^{2}\left(C_{o x}+C_{s i}\right) C_{s i} R_{s i}^{2}}{1+\omega^{2}\left(C_{o x}+C_{s i}\right)^{2} R_{s i}^{2}} \\
R_{s u b} & =\frac{1}{\omega^{2} C_{o x}^{2} R_{s i}}+\frac{R_{s i}\left(C_{o x}+C_{s i}\right)^{2}}{C_{o x}^{2}}
\end{aligned}
$$

$C_{O x}$ is the capacitance between substrate and spiral structure and is given by

$$
C_{o x}=\frac{1}{2} \times l \times W \frac{\epsilon_{o x}}{t_{o x}}
$$

The resistance $R_{s i}$ and capacitance $C_{s i}$ are the parasitics of the silicon substrate.

$$
\begin{aligned}
R_{s i} & =\frac{2}{l W G_{u a}} \\
C_{s i} & =\frac{1}{2} l W C_{u a}
\end{aligned}
$$

where $C_{u a}$ and $G_{u a}$ are the unit area conductance and capacitance.

\section{A. Figures of merit - Quality factor}

$$
\begin{aligned}
Q= & \frac{\omega L}{R_{s}} \times \frac{R_{\text {sub }}}{R_{s u b}+\left[\left(\frac{\omega L}{R_{s}}\right)^{2}+1\right] R_{s}} \\
& \times\left[1-\frac{R_{s}^{2}\left(C_{s u b}+C_{L}\right)}{L}-\omega^{2} L\left(C_{s u b}+C_{L}\right)\right]
\end{aligned}
$$

The value of $\mathrm{Q}$ is critical because it determines the self resonant frequency (where $\mathrm{Q}$ is zero) and maximum frequency (where $\mathrm{Q}$ is maximum).

\section{OPTIMIZATION OF SPIRAL INDUCTOR USING ELECTROMAGNETIC SIMULATOR}

The spiral inductor with the geometric parameters discussed in the previous section is simulated using an electromagnetic simulator. SONNET is a well known EM simulator which is used to design the spiral layout by giving the dimensions as input. Once the geometrical parameters are fed, the spiral assistant tool will give the inductance value. All the parasitic elements can be extracted by importing the design from the spiral inductor assistant to SONNET.

Figure 10 to Figure 15 shows the extracted parameters for millimeter wave frequency ranging from $30 \mathrm{GHz}$ to $70 \mathrm{GHz}$. The value of the parasitics at $60 \mathrm{GHz}$ is determined as in Table I and used for the design of a millimeter wave oscillator[10].

TABLE I

INDUCTOR EXTRACTED PARAMETER VALUES AT $60 \mathrm{GHZ}$

\begin{tabular}{cc}
\hline Parameters & Extracted values \\
\hline $\mathrm{L}$ & $154 \mathrm{pH}$ \\
$R_{s}$ & $1.76 \mathrm{ohms}$ \\
$C_{\text {sub }}$ & $5.8 \mathrm{fF}$ \\
$R_{\text {sub }}$ & $27.7 \mathrm{ohms}$ \\
$C_{o x}$ & $4 \mathrm{fF}$ \\
\hline
\end{tabular}


INTERNATIONAL JOURNAL OF CIRCUITS, SYSTEMS AND SIGNAL PROCESSING

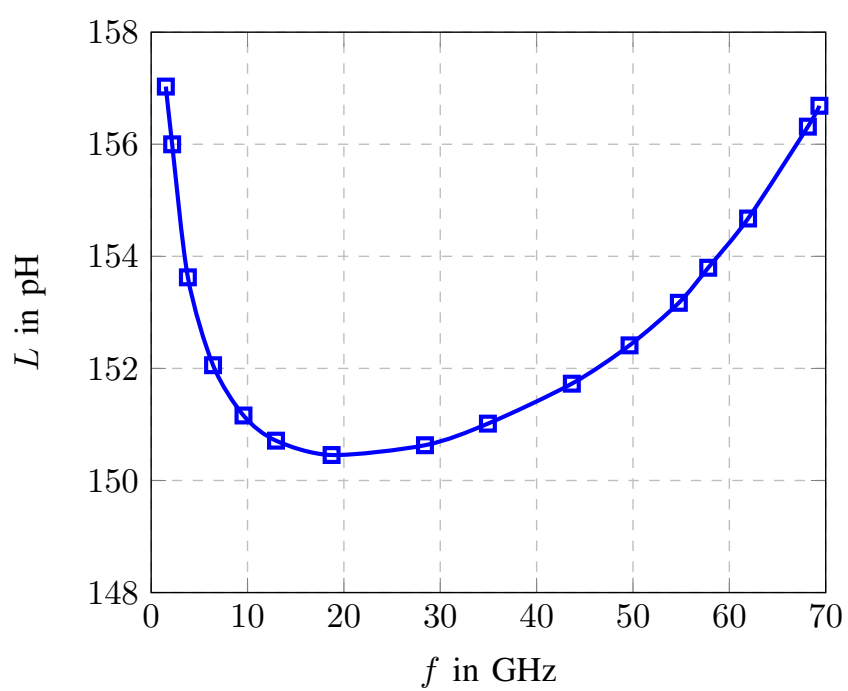

Fig. 10. Inductance versus frequency

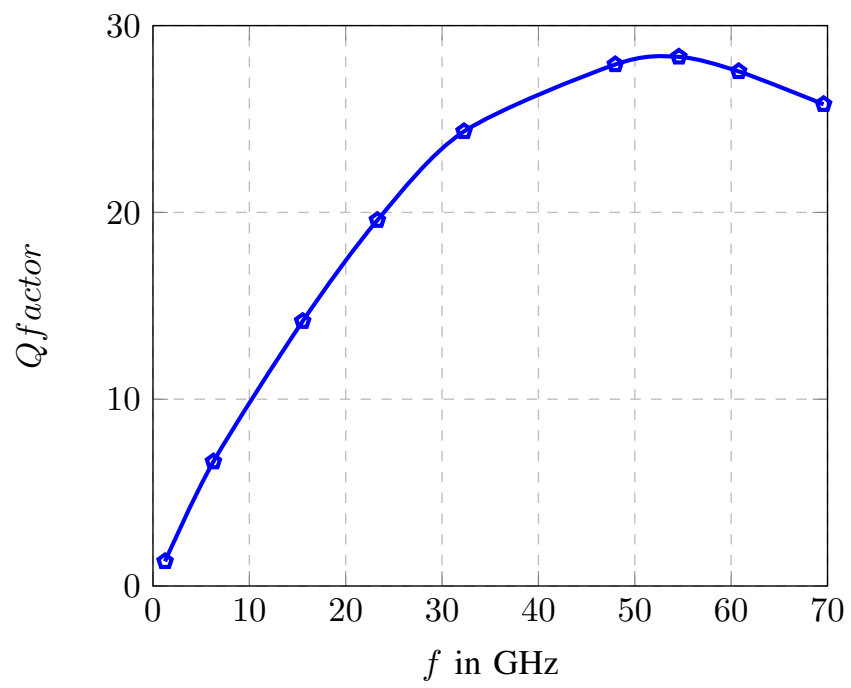

Fig. 11. Q factor versus frequency

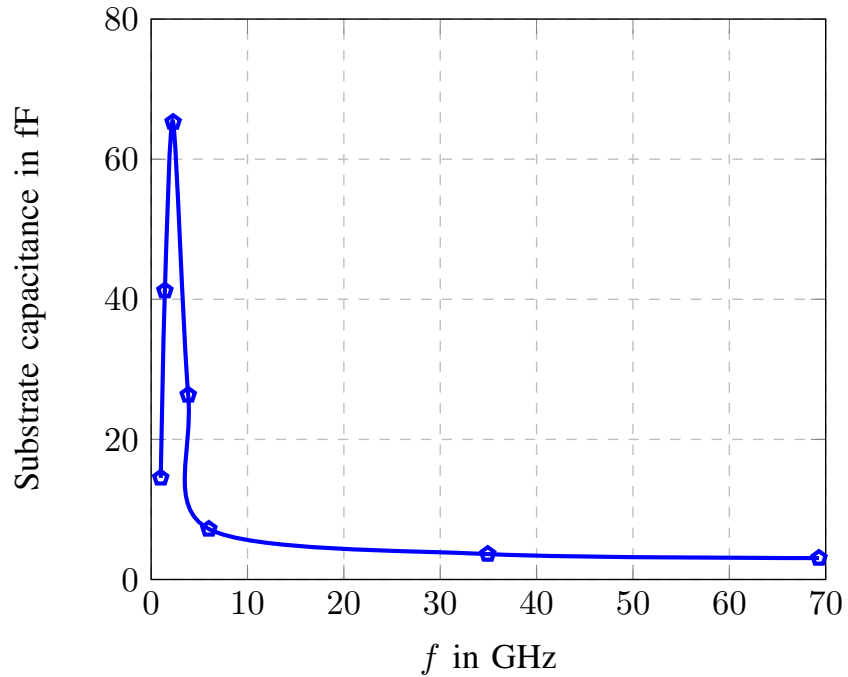

Fig. 14. $C_{\text {sub }}$ versus frequency

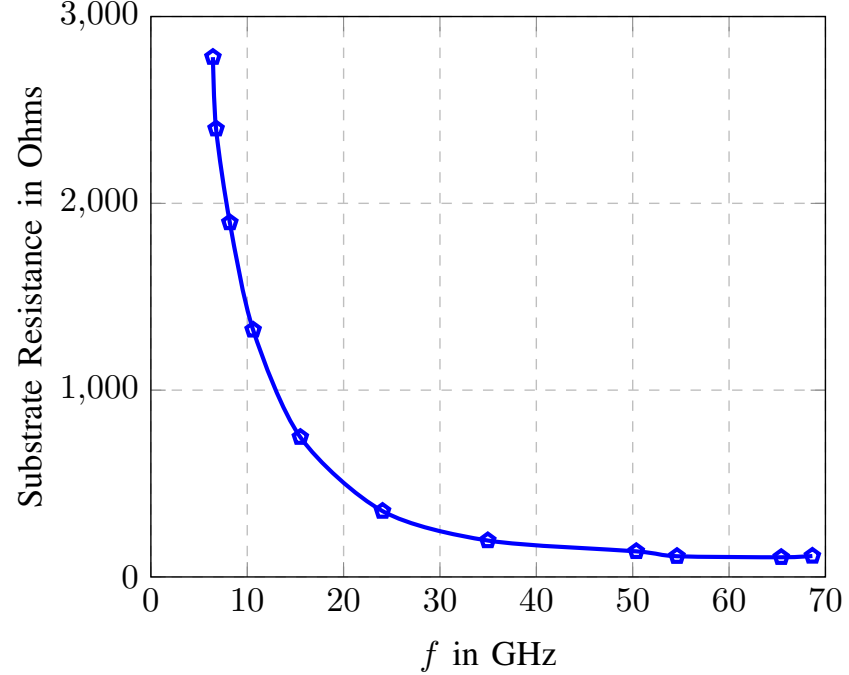

Fig. 12. $R_{\text {sub }}$ versus frequency

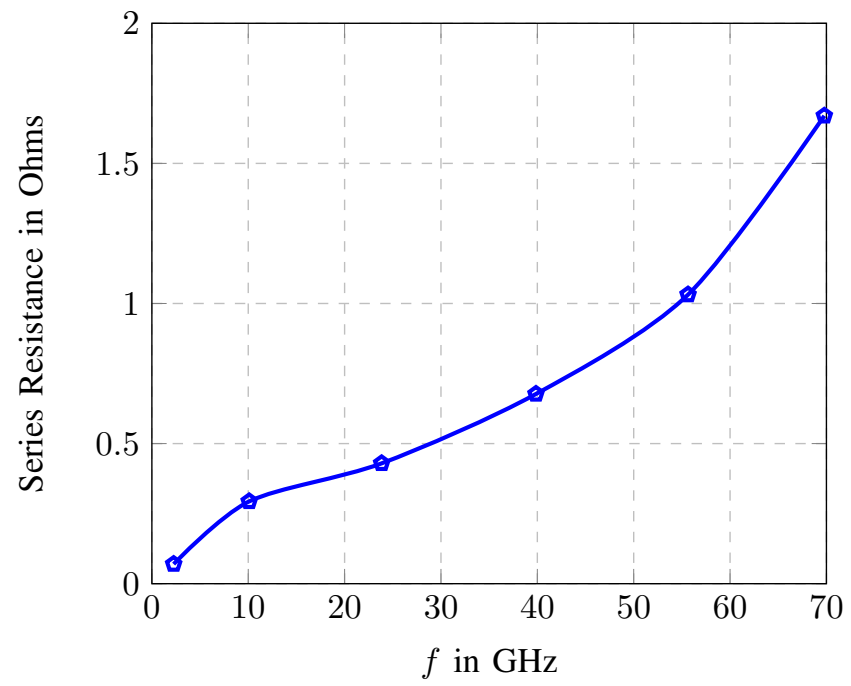

Fig. 13. $R_{s}$ versus frequency

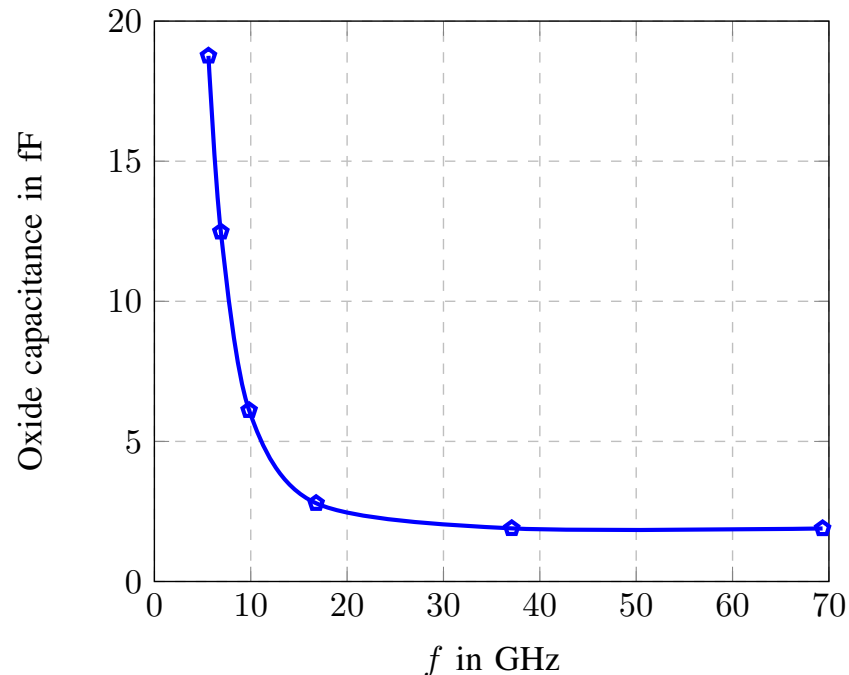

Fig. 15. $C_{o x}$ versus frequency 


\section{DESIGN OF MILLIMETER WAVE OSCILLATOR USING EXTRACTED PARAMETERS OF THE INDUCTOR}

The inductor designed using the EM simulator shows many parasitic elements and this may lead to a very low quality factor even though it shows a high $\mathrm{Q}$ at this stage. This lowering of $\mathrm{Q}$ is primarily due to the losses like ohmic loss and loss in the substrate [11].

The most important component in an LC based oscillator is the inductor because it determines the $\mathrm{Q}$ of the tank and if the $\mathrm{Q}$ is small, it may lead to increased phase noise which is not preferred. So maintaining the $\mathrm{Q}$ factor is extremely important and crucial.

The oscillator was designed with a power constraint of 10 $\mathrm{mW}$ from a supply voltage of $1 \mathrm{~V}$.

The bias current is selected based on $\frac{g_{m}}{I_{D}}$ methodology.

$$
\begin{gathered}
I_{D} \leq I_{\text {bias }} \leq 4.75 \mathrm{~mA} \\
\frac{g_{m}}{I_{D}}=5 \\
I_{D} \times R_{p} \geq V_{\text {out }} \\
\therefore R_{p} \geq \frac{V_{\text {out }}}{I_{D}}
\end{gathered}
$$

Finally $R_{p}$ can be obtained as

$$
R_{p}=Q^{2} \times R_{s}
$$

TABLE II

Simulation Results of Millimeter waVe oscillator at $60 \mathrm{GHZ}$

\begin{tabular}{ccc}
\hline Design parameters & Target values & Simulated values \\
\hline Center frequency & $60 \mathrm{GHz}$ & $59.48 \mathrm{GHz}$ \\
Maximum Phase noise & $<-85 \mathrm{dBc} / \mathrm{Hz}$ & $-87 \mathrm{dBc} / \mathrm{Hz}$ \\
Minimum output Swing & $\approx V_{D D}$ & $1.61 V_{p p}$ \\
Maximum power & $10 \mathrm{~mW}$ & $9.62 \mathrm{~mW}$ \\
\hline
\end{tabular}

\section{CONCLUSION}

This paper presents the design of a spiral inductor for millimeter wave oscillator. The inductor is first designed using an optimization algorithm, the results were taken to simulate in an electromagnetic simulator. The parasitic values were extracted from the EM simulator. Using the extracted values , an oscillator is designed at millimeter wave frequency at 59.48 GHz. The simulated inductance has a value of $154 \mathrm{pH}$ which was suitable for MMW region with a high $\mathrm{Q}$ factor of more than 20. But this $\mathrm{Q}$ factor has been deteriorated to a lower value close to 13 , during the circuit level simulations due to the losses in the substrate and the ohmic losses. The designed oscillator has a reasonable phase noise of $-87 \mathrm{dBc} / \mathrm{Hz}$ at 59.48 $\mathrm{GHz}$ consuming a power of $9.62 \mathrm{~mW}$.

\section{REFERENCES}

[1] Cao, Seok, E. and O, K. K. Millimeter-wave CMOS voltage-controlled oscillators, 2007 IEEE Radio and Wireless Symposium 2007.

[2] M.Tiebout, Low Power VCO Design in CMOS, Springer, 2006.

[3] Jean Olivier Plouchart, J. K. A $31 \mathrm{GHz}$ CML ring vco with 5.4 ps delay in a 0.12 um SOI CMOS technology, Proceedings of the 29th European Solid-State Circuits Conference, 2003.

[4] Marius Voicu1,Domenico Pepe, and Domenico Zito Performances and Trends in Millimeter-Wave CMOS Voltage Controlled Oscillators, 19th IEEE International Conference on Electronics, Circuits and Systems (ICECS, 2012

[5] Razavi, B, RF Microelectronics, 3rd ed. Pearson Education, 2012.

[6] Nithin M, Harish M Kittur Phase locking approaches in millimeter wave frequency synthesizers - Design overview of charge pump phase locked loops, WSEAS Transactions on Circuits and Systems,2020.

[7] Kenichi Okada and Kazuya Masu, Modeling of Spiral Inductors, Tokyo Institute of Technology,2010.

[8] Genemala Haobijam, Roy Paily Palathinkal, Design and Analysis of Spiral Inductors, Springer,2014.

[9] Kouznetsov, K. and Meyer, Phase noise in LC oscillators, IEEE Journal of Solid-State Circuits 35(8), 1244-1248. 2000.

[10] Marco Vigilante, P. R, $5 G$ and E-Band Communication Circuits in DeepScaled CMOS, Analog Circuits and Signal Processing, Springer 2018.

[11] Nithin, M., Ramesh, S. and Kittur, H. M Design of millimeter wave LC oscillators for $5 \mathrm{~g}$ applications, International Conference on Communication and Signal Processing (ICCSP)', IEEE. 2019.

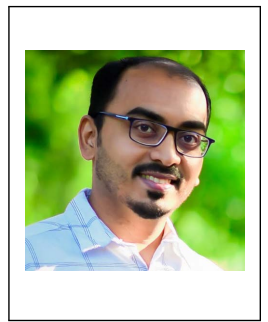

Nithin M (S'20) received the B.Tech degree in Electronics and Communication Engineering in 2008 from Mohandas College of Engineering and Technology, Trivandrum,University of Kerala, and the M.Tech degree in VLSI Design from Vellore Institute of Technology, Vellore in 2014. He is pursuing Ph.D. degree in Analog-Radio Frequency Integrated Circuits Design from Vellore Institute of Technology, Vellore, Tamil Nadu, India.

He is currently Assistant Professor in the Department of ECE at RV College of Engineering, Bengaluru, Karnataka, India. His research interests include the Analog Integrated circuits,High frequency oscillators and RF Integrated Circuits.

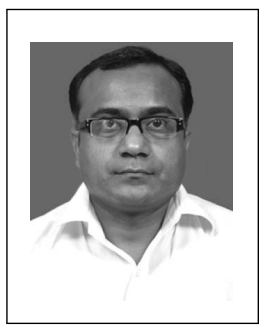

Harish M. Kittur (M'10) received the B.Sc. degree in physics, mathematics, and electronics from Karnataka University, Dharwad, India, the M.Sc. degree in physics from IIT Bombay, Mumbai, India, the M.Tech. degree in solid-state technology from IIT Madras, Chennai, India, and the Ph.D. degree from RWTH Aachen University, Aachen, Germany.

$\mathrm{He}$ is currently Professor and Dean of the School of Electronics Engineering with VIT, Vellore, India. His current research interests include low-power VLSI design, memory design, and nanoelectronics.,Dr. Kittur is a Life Member of the Institution of Electronics and Telecommunication Engineers and a member of the Institution of Engineering and Technology. 